\title{
The inability to ignore auditory distractors as a function of visual task perceptual load
}

\author{
DONALD J. TELLINGHUISEN and ERIN J. NOWAK \\ Calvin College, Grand Rapids, Michigan
}

\begin{abstract}
Using a response competition paradigm, we investigated the ability to ignore target responsecompatible, target response-incompatible, and neutral visual and auditory distractors presented during a visual search task. The perceptual load model of attention (e.g., Lavie \& Tsal, 1994) states that task-relevant processing load determines irrelevant distractor processing in such a way that increasing processing load prevents distractor processing. In three experiments, participants searched sets of one (easy search) or six (hard search) similar items. In Experiment 1, visual distractors influenced reaction time (RT) and accuracy only for easy searches, following the perceptual load model. Surprisingly, auditory distractors yielded larger distractor compatibility effects (median RT for incompatible trials minus median RT for compatible trials) for hard searches than for easy searches. In Experiments 2 and 3, during hard searches, consistent RT benefits with response-compatible and RT costs with response-incompatible auditory distractors occurred only for hard searches. We suggest that auditory distractors are processed regardless of visual perceptual load but that the ability to inhibit cross-modal influence from auditory distractors is reduced under high visual load.
\end{abstract}

When searching for a target item in a complex environment, an organism must attend to relevant stimuli while avoiding distraction by irrelevant stimuli. This ability to ignore irrelevant information is vital to efficient target detection. Lavie (e.g., Lavie, 1995; Lavie \& Cox, 1997; Lavie \& Tsal, 1994) has identified difficulty of the target search, or perceptual load, as a determining factor in how irrelevant information influences search efficiency. Lavie's perceptual load model assumes that perception is a limited capacity mechanism but that processing occurs automatically. Hence, the perceptual load incurred in processing relevant stimuli directly determines the extent to which irrelevant information is also processed. Specifically, if all of an organism's processing capacity is utilized in processing relevant stimuli, no additional processing capacity is available, and irrelevant stimuli will not be processed. Therefore, distractors do not interfere with high perceptual load tasks.

The type of task typically utilized to demonstrate the perceptual load model is a modification of Eriksen and Eriksen's (1974) visual response competition paradigm. Lavie and Cox (1997), for example, required participants to

Portions of these data were presented at the 42nd Annual Meeting of the Psychonomic Society, November 2001, in Orlando, FL. This research was supported in part by a Research Fellowship awarded to the first author and was funded by the Calvin College Board of Trustees and by a McGregor Fellowship granted to the second author. The authors thank Laura Luchies, Emily Schemper, and Melissa Wiersum for their help in data collection and Lisa Fournier and two anonymous reviewers for helpful comments on an earlier draft of this paper. Correspondence concerning this article should be addressed to D. J. Tellinghuisen, Department of Psychology, Calvin College, Grand Rapids, MI 49546 (e-mail: dtelling@calvin.edu). search several centrally displayed letters for one of two target letters $(\mathrm{X}$ or $\mathrm{N})$ while ignoring peripherally presented irrelevant distractors. Perceptual load was manipulated by varying the similarity between targets and nontargets. In the high-load (hard search) condition, nontargets were similar to the targets (e.g., M, K, or Z), whereas in the low-load (easy search) condition, nontargets were dissimilar to the targets (e.g., O). Irrelevant visual distractors influenced reaction times (RTs) for identifying the target in the easy search condition, but not in the hard search condition. Specifically, the presence of incompatible distractors (stimuli that required the response opposite the target response, such as an X when the target was an $\mathrm{N}$ ) resulted in longer RTs than did compatible distractors (stimuli that required the same response as the target response, such as an $\mathrm{X}$ when the target was an $\mathrm{X}$ ) or neutral distractors (stimuli with no response associations, such as a $\mathrm{T}$ when the target was an X). In line with the perceptual load model, Lavie and Cox concluded that under the high loads of relevant processing that occurred in the hard search task, available processing capacity was exhausted. In this case, no spare capacity remained to process irrelevant stimuli, so these stimuli had no influence on RTs. Under the lower processing loads of the easy search task, however, capacity was not exhausted. In this case, spare processing capacity automatically spilled over to irrelevant distractors, resulting in their having an influence on RTs. The perceptual load model assumes that all of attentional capacity is utilized at all times (Lavie \& Tsal, 1994). Although an individual can prioritize attentional allocation, any unallocated capacity is automatically allocated to other stimuli. This assumption makes the load model markedly different from depic- 
tions of attentional capacity as being more flexible and controlled by an individual's volition (e.g., Kahneman, 1973; Navon \& Gopher, 1979). The load model, therefore, assumes that irrelevant information will be excluded from processing only if processing prioritized as relevant exhausts all available capacity.

Lavie and Tsal (1994) stated that perceptual load is conceptualized as the number of units in the display, as well as the complexity of the processing required for each unit. Units are the number of items that constitute different alternatives for the relevant responses in a task. Hence, a low-load task is one that includes few different items and/or requires little processing per unit. Although Lavie has primarily used number of units or display size to manipulate level of perceptual load (e.g., Lavie, 1995; Lavie \& Cox, 1997), others have varied the display quality of relevant targets to manipulate perceptual load (e.g., Handy \& Mangun, 2000; Handy, Soltani, \& Mangun, 2001) to yield similar results. Although Lavie (e.g., Lavie $\&$ Tsal, 1994) has stated that physical distinctiveness, eccentricity, and stimulus onset asynchrony are important in determining processing priority of relevant information, such factors influence only the magnitude of distractor effects; they cannot entirely prevent processing of irrelevant information. The perceptual load model states that capacity limitations serve as the final determinant of selective attention unless the distractor is degraded, has little or no association with the relevant response, or appears after a response has been generated (Lavie \& Tsal, 1994).

An issue regarding the perceptual load model that has not been substantially addressed is whether it holds across modalities. Studies on the effects of irrelevant information during a selective attention task have primarily used visual stimuli (e.g., Eriksen \& Eriksen, 1974; Lavie, 1995), whereas only a few studies have used auditory stimuli (e.g., Moray, 1959; Zelniker, 1971). The question of how processing load influences attentional allocation across modalities, such as vision and audition, is theoretically and pragmatically important. Wickens (1980), among others (see Duncan, Martens, \& Ward, 1997 , for a more recent example), has suggested that auditory and visual processing resources or capacity may be somewhat or completely separate. Kahneman (1973), however, has asserted that limitations on processing capacity are central, rather than modality specific. Finally, others, such as Driver and Spence (1998), have demonstrated extensive cross-modal links in attention across several modalities, including audition and vision. Driver and Spence, in their review of cross-modal attention, indicated that although selective attention to a particular modality is possible, attention across modalities is coordinated when the information being attended comes from a common spatial location.

How capacity is distributed has obvious implications for the perceptual load model. If separate attentional capacities exist for different modalities, it follows that exhausting visual processing capacity would have little implication for how auditory processing capacity is allocated. If, however, attentional capacity is centrally limited, exhausting capacity in one modality should restrict attention allocation to another modality. Finally, if capacity is linked across modalities, exhausting one modality's capacity should impact processing in another modality, particularly as processing load increases. Therefore, the outcome of cross-modal studies of attention in which perceptual load is manipulated could inform basic theories of both cross-modal attention and attentional capacity.

One recent study has explored the intersection between processing load and cross-modal attention. Rees, Frith, and Lavie (2001) evaluated the perception of irrelevant visual motion as a function of auditory perceptual load. Participants monitored identical streams of spoken words and in the easy, or low-load, condition, detected words spoken in a loud voice among words spoken in a quiet voice or, in the high-load condition, detected bisyllabic words among monosyllabic and trisyllabic words. The visual stimuli consisted of 400 white dots. The dots appeared either static or moving radially away from a central fixation point at a constant velocity, with dots leaving the edge of the viewing screen replotted at the screen's center to maintain a constant dot density. Using positron emission tomography (PET) in their Experiment 1, Rees et al. (2001) compared the amount of activity in motion-related visual areas under both conditions of load when the dots were static or in motion. Significant PET activation was found for motion, when compared with no motion, for both the low- and the high-load conditions, particularly in areas V5, V3a, and the kinetic occipital area. In Experiment 2 of Rees et al. (2001), participants viewed only presentations of moving dots while performing the same auditory load tasks as those in Experiment 1. After monitoring and responding to a 60 -sec sequence of auditory words, the participants indicated the duration of the perceived motion aftereffect (MAE) by pressing a button. MAE duration was not influenced by auditory perceptual load.

These results contrast with those of Experiment 3 of Rees et al. (2001), which included data that were reported in part by Rees, Frith, and Lavie (1997). Stimuli and tasks in those experiments were similar to those in Experiments 1 and 2 of Rees et al. (2001), with the major difference that the linguistic target stimuli were visual rather than auditory. Using functional magnetic resonance imaging (fMRI), Rees et al. (1997) found activation in visual areas in relation to the presence of irrelevant motion in the low-load condition (detecting words presented in uppercase rather than in lowercase letters), but not in the high-load condition (detecting words that were bisyllabic). In addition, Rees et al. (2001, Experiment 3) reported that MAE duration was significantly longer following the low-load task than following the high-load task. Handy et al. (2001) obtained similar results when perceptual load was varied by manipulating the visual clarity (and hence, the visuocortical processing) of a target presented along with visual distractors. 
They found that incompatible distractors presented in a high perceptual load condition yielded lower P1 eventrelated potential activity than did those in a low perceptual load condition. Together with Lavie's other research (1995, 1997; Lavie \& Cox, 1997), these studies indicate that the perceptual load model holds when the distractors and the target stimuli are both visual, but not when the distractors are visual but the target stimuli are auditory. Perception of distractors presented in another modality, however, is not influenced by perceptual load (Rees et al., 2001). Thus, Rees et al. (2001) suggested that attentional capacity is modality specific.

The methods of Rees et al. (2001), however, differ in important ways from Lavie's other tests of the perceptual load model (Lavie, 1995, 1997; Lavie \& Cox, 1997), in which load effects were measured in the context of a response competition paradigm. In those studies, the distractors could be associated with the same response as the target (compatible) or with the response opposite that required for the target (incompatible). The change in target task performance was measured as a function of task load and distractor compatibility. It is significant that Rees et al. (2001) focused on whether distractor perception per se was influenced by processing load. In Rees et al.'s (2001) research, this is the most reasonable measure of distractor effects, since their distractors were neutral with respect to the target task; the distractors were unrelated to responses for the primary task. Distractor stimuli should not have been expected to impact performance of the primary task, and the authors did not report any attempt to measure such an influence. Thus, the tasks used by Rees et al. (2001) could measure cross-modal perception of distractor stimuli but could not quantify cross-modal interference caused by distractor stimuli.

How might responses to targets in one modality be affected if distractors are compatible or incompatible with the target response but presented in another modality? In the present research, we sought to determine the degree to which cross-modal interference is influenced by perceptual load. We utilized the method of Lavie and Cox (1997, Experiment 1) and extended it by including auditory distractors as well as visual ones. This method allowed us to present to-be-ignored stimuli that should interfere more directly with the target task, because the distractors had a direct relationship to performance of the target task. In addition to this methodological difference with Rees et al. (2001), also note that we employed a visual search task performed with simultaneous auditory distractors, rather than an auditory detection task performed with simultaneous visual distractors.

The perceptual load model (Lavie \& Tsal, 1994) assumes that perception is automatic and involuntary but that it has limited capacity. If the capacity limitation for the processing of stimuli from one modality is independent of the capacity limitation for the processing of stimuli from another modality, as Rees et al. (2001) concluded, task-incompatible information presented in one modality should significantly interfere with processing in another modality regardless of task load. We expected interference from incompatible auditory distractors in the present study, because these distractors were related to responses for the primary task, unlike the distractors of Rees et al. (2001). Therefore, we hypothesized that cross-modal distractor rejection should not vary as a function of target load. Significantly longer RTs and lower accuracy rates for incompatible than for compatible distractors should be found for both low- and high-load searches. When the target array and the distractor are both presented in the same modality, however, distractor rejection should occur under high-load conditions, but not under low-load conditions, as Lavie and Cox (1997) found.

\section{EXPERIMENT 1}

\section{Method}

Participants. Thirty undergraduate students (15 females, 15 males) from a midwest liberal arts college participated on a volunteer basis. Their mean age was 20.1 years $(S D=1.5)$. All the participants had normal or corrected-to-normal near-vision acuity, as measured with a Snellen eye chart.

Apparatus and Stimuli. The participant was seated at a table in front of an Apple iMac computer in a normally lit room. The participant's viewing distance was fixed at $57 \mathrm{~cm}$, using a chinrest. The stimuli were presented using Super Lab Pro (Cedrus, 1998) software. An Apple iMac keyboard was used to record responses. Visual stimuli were presented on the iMac 15-in. color monitor. Auditory stimuli were presented via headphones (Koss Model TD/65). All of the letters were white, presented in uppercase Helvetica font on a black background.

The visual stimuli were similar to those used by Lavie and Cox (1997) and are depicted in Figure 1. The target letter, either X or N, appeared randomly and an equal number of times in one of six positions arranged in a circular pattern with a radius of $2.1^{\circ}$ from a central fixation point. The target letters subtended a visual angle of $0.6^{\circ}$ vertically and $0.4^{\circ}$ horizontally. The distractor letter was pre-

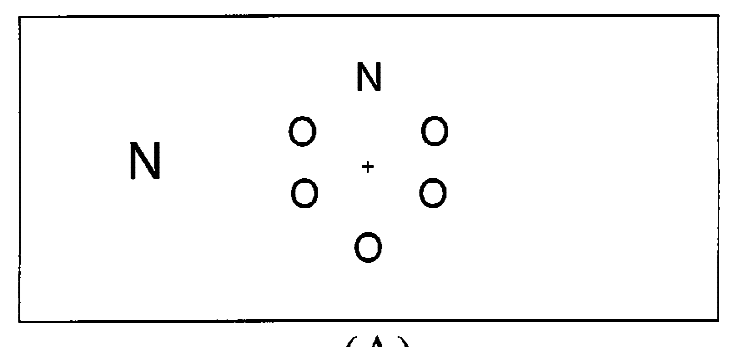

\section{(A)}

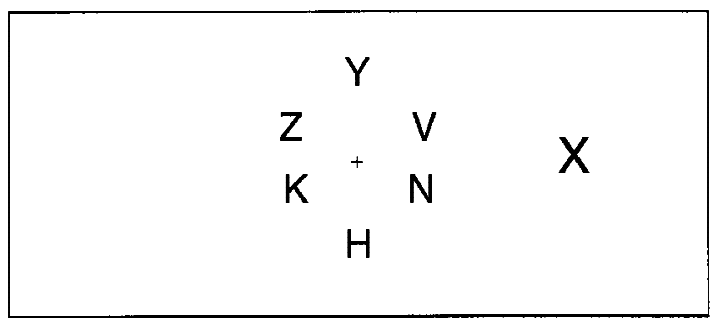

(B)

Figure 1. Examples of (A) an easy search condition with a compatible visual distractor and $(B)$ a hard search condition with an incompatible visual distractor. 
sented to the right or the left of the circular display, $4.3^{\circ}$ from the central fixation point. The distractor letter subtended a visual angle of $0.9^{\circ}$ vertically and $0.5^{\circ}$ horizontally.

Auditory distractor stimuli were presented in a female voice. The participants heard a single letter spoken simultaneously with the presentation of the visual stimuli. The decibel levels and the duration of the auditory stimuli were about as follows: $\mathrm{L}=69 \mathrm{~dB}, 310 \mathrm{msec}$; $\mathrm{T}=65 \mathrm{~dB}, 300 \mathrm{msec} ; \mathrm{N}=72 \mathrm{~dB}, 300 \mathrm{msec}$; and $\mathrm{X}=70 \mathrm{~dB}$, $380 \mathrm{msec}$. Decibel readings were based on measurements taken with a Radio Shack sound-level meter held approximately $1 \mathrm{~cm}$ away from the headphone speaker. The durations of the auditory stimuli were the minimum needed to include all portions of the vocalization of each letter necessary to make it comprehensible.

Design. This experiment employed a fully randomized withinsubjects design. The independent variables included task difficulty (easy or hard), distractor modality (visual or auditory), and distractor compatibility (compatible, incompatible, or neutral). All the independent variables occurred randomly and in equal numbers for each participant. The dependent variables were median RT and error rate. RTs were defined as the duration between the onset of the target and distractor stimuli and the participant's keyboard response.

In the easy condition, the letter $\mathrm{O}$ appeared in five positions, and the target letter occupied the sixth position. In the hard condition, the letters $\mathrm{H}, \mathrm{Y}, \mathrm{Z}, \mathrm{K}$, and $\mathrm{V}$ appeared randomly in five positions of the circle, and the target letter appeared in the sixth position. In both conditions, target position was randomized.

A visual distractor was one that appeared on the computer screen, either to the right or to the left of the circular display. An auditory distractor was one that was presented via the headphones. Half of the trials included a visual distractor, and half included an auditory distractor.

The letters N, X, L, and T were used as the distractor letters. Each of the distractor letters was evenly divided among compatible, incompatible, or neutral conditions. Compatible distractors were identical to the target letter in that particular trial. Incompatible distractors were the opposite target letter (X when the target was $\mathrm{N}$ and vice versa). Neutral distractors were the letter $\mathrm{L}$ for one half of the neutral trials and the letter $\mathrm{T}$ for the other half.

Procedure. The participants were tested individually in a small room. Near-vision acuity was measured with a Snellen 20 -ft. equivalent eye chart prior to the experiment. The participants were seated at a desk and were instructed to keep their heads in a chinrest for the duration of the experiment. The participants were then orally instructed to ignore the distractor letters when searching for the target letter. Instructions were again given on the computer screen before the experiment began.

Each display began with a $100-$ msec beep $(66 \mathrm{~dB})$ followed by a fixation screen, which appeared for $1 \mathrm{sec}$. The fixation screen consisted of a + mark in the center of the screen. The participants were instructed to focus on this point throughout the experiment. The target display then appeared for $100 \mathrm{msec}$. Onset of distractors, whether visual or auditory, was simultaneous with the target display onset. The participants rested their left index fingers on the $\mathrm{X}$ key and their right index fingers on the $\mathrm{N}$ key. The participants were instructed to press the $\mathrm{X}$ key or the $\mathrm{N}$ key to indicate which target letter was presented. Speed and accuracy were both emphasized.

Three blocks of 144 trials each were presented. The participants were given 2-min breaks between blocks. The participants were told that Block 1 was practice, and the data from that block were not analyzed. The data from Blocks 2 and 3 were used for data analysis. This yielded 24 presentations per condition.

\section{Results}

The results show significant distractor compatibility effects for visual distractors in the easy search conditions and for auditory distractors in both the easy and the hard search conditions.
We computed median correct RTs and percentage error rates for each participant as a function of distractor modality (auditory or visual), search difficulty (easy or hard), and distractor compatibility (compatible, incompatible, or neutral). Response latencies below $100 \mathrm{msec}$ or above $2 \mathrm{sec}$ were counted as errors.

We performed a repeated measures analysis of variance (ANOVA) on the error rate data with three factors: modality (2) $\times$ difficulty $(2) \times$ compatibility (3). These data are shown in Table 1. Significant main effects of difficulty $[F(1,29)=173.83, p<.001]$ and of compatibility $[F(2,58)=11.16, p<.001]$ and a significant modality $\times$ compatibility interaction $[F(2,58)=11.72$, $p<.001]$ were subsumed in a significant modality $\times$ difficulty $\times$ compatibility interaction $[F(2,58)=5.16, p<$ .009]. On the whole, the participants had higher error rates for hard searches than for easy searches, with the exception that the error rate for the auditory, hard, compatible condition did not significantly differ from that for the visual, easy, incompatible condition. In addition, the visual, easy, incompatible condition yielded an error rate that was higher than the auditory, easy, compatible error rate. Finally, the auditory, hard, compatible condition error rate was lower than the error rates for all other hard conditions

We also performed a repeated measures ANOVA on the RT data that are presented in Table 1. Modality (2), difficulty (2), and compatibility (3) were once again factors. The three-way modality $\times$ difficulty $\times$ compatibility interaction was significant $[F(2,34)=7.71, p<.002]$. The differences that produced this significant interaction are central to understanding these influences. The question of interest in this research is how distractor compatibility influences RT, particularly as a function of distractor modality. This question can best be addressed by exploring the magnitude of the differences between RTs to trials with neutral distractors and those to trials with either compatible or incompatible distractors. Specifically, it is of most interest to determine whether the magnitude of these differences is greater than zero. Nonzero differences would indicate an effect of distractor compatibility. Following the data analysis strategy of Lavie and Cox (1997), we computed three new dependent variables for each combination of modality and difficulty. Benefits were computed for each participant by subtracting the median RT for compatible trials from the median RT for neutral trials, yielding a measure of the RT benefit, relative to baseline, of being presented with a distractor that represented the same response as that required for the target. Costs were computed by subtracting the median RT for neutral trials from the median RT for incompatible trials, yielding a measure of the RT cost, relative to baseline, of being presented with a distractor that represented the response opposite that required for the target. Compatibility effects were computed by subtracting the median RT for compatible trials from the median RT for incompatible trials, yielding a measure of the overall difference in RT due to distractor compatibility. Mean costs and benefits are presented in Figure 2, and 
Table 1

Median Reaction Times (RTs, in Milliseconds) and Error Rates (as Percentages), With 95\% Confidence Intervals (CIs), as a Function of Distractor Type (Modality and Compatibility) and Search Difficulty for Experiments 1-3

\begin{tabular}{|c|c|c|c|c|c|c|c|c|c|}
\hline \multirow[b]{3}{*}{ Modality } & \multirow[b]{3}{*}{ Compatibility } & \multicolumn{4}{|c|}{ Easy Search } & \multicolumn{4}{|c|}{ Hard Search } \\
\hline & & \multicolumn{2}{|r|}{ RT } & \multicolumn{2}{|c|}{ Error } & \multicolumn{2}{|r|}{ RT } & \multicolumn{2}{|c|}{ Error } \\
\hline & & Med. & $\mathrm{CI}$ & Med. & CI & Med. & CI & Med. & $\mathrm{CI}$ \\
\hline \multicolumn{10}{|c|}{ Experiment 1} \\
\hline Visual & $\begin{array}{l}\text { Compatible } \\
\text { Neutral } \\
\text { Incompatible }\end{array}$ & $\begin{array}{l}579 \\
580 \\
615\end{array}$ & $\begin{array}{l}533-614 \\
547-612 \\
583-548\end{array}$ & $\begin{array}{l}4.2 \\
3.9 \\
6.9\end{array}$ & $\begin{array}{l}1.9-6.5 \\
1.8-6.0 \\
4.2-9.7\end{array}$ & $\begin{array}{l}755 \\
727 \\
747\end{array}$ & $\begin{array}{l}711-799 \\
678-776 \\
702-793\end{array}$ & $\begin{array}{l}18.4 \\
15.9 \\
20.5\end{array}$ & $\begin{array}{l}14.9-21.9 \\
12.0-19.8 \\
16.6-24.4\end{array}$ \\
\hline Auditory & $\begin{array}{l}\text { Compatible } \\
\text { Neutral } \\
\text { Incompatible }\end{array}$ & $\begin{array}{l}550 \\
569 \\
572\end{array}$ & $\begin{array}{l}521-579 \\
540-597 \\
544-601\end{array}$ & $\begin{array}{l}2.6 \\
4.5 \\
4.8\end{array}$ & $\begin{array}{l}1.2-3.9 \\
2.7-6.4 \\
2.1-7.4\end{array}$ & $\begin{array}{l}692 \\
767 \\
789\end{array}$ & $\begin{array}{l}659-726 \\
715-819 \\
734-843\end{array}$ & $\begin{array}{l}11.9 \\
22.1 \\
19.6\end{array}$ & $\begin{array}{r}9.3-14.5 \\
18.8-25.3 \\
16.0-23.3\end{array}$ \\
\hline \multicolumn{10}{|c|}{ Experiment 2} \\
\hline Auditory & $\begin{array}{l}\text { Compatible } \\
\text { Neutral } \\
\text { Incompatible } \\
\text { No distractor }\end{array}$ & $\begin{array}{l}557 \\
567 \\
575 \\
552\end{array}$ & $\begin{array}{l}518-597 \\
528-605 \\
534-616 \\
515-588\end{array}$ & $\begin{array}{l}2.3 \\
1.7 \\
3.3 \\
3.3\end{array}$ & $\begin{array}{l}1.1-3.4 \\
0.7-2.8 \\
1.4-5.2 \\
1.8-4.8\end{array}$ & $\begin{array}{l}682 \\
723 \\
756 \\
713\end{array}$ & $\begin{array}{l}628-735 \\
671-775 \\
700-811 \\
650-775\end{array}$ & $\begin{array}{r}9.0 \\
12.0 \\
27.1 \\
15.6\end{array}$ & $\begin{array}{r}6.2-11.8 \\
8.7-15.4 \\
21.9-32.3 \\
11.7-19.5\end{array}$ \\
\hline \multicolumn{10}{|c|}{ Experiment 3} \\
\hline Auditory & $\begin{array}{l}\text { Compatible } \\
\text { Neutral } \\
\text { Incompatible } \\
\text { White noise }\end{array}$ & $\begin{array}{l}550 \\
558 \\
572 \\
547\end{array}$ & $\begin{array}{l}518-583 \\
528-588 \\
541-603 \\
519-575\end{array}$ & $\begin{array}{l}3.8 \\
2.7 \\
3.0 \\
4.0\end{array}$ & $\begin{array}{l}2.1-5.5 \\
1.2-4.2 \\
1.6-4.4 \\
2.0-5.9\end{array}$ & $\begin{array}{l}693 \\
772 \\
818 \\
760\end{array}$ & $\begin{array}{l}654-732 \\
717-827 \\
765-871 \\
712-808\end{array}$ & $\begin{array}{l}12.4 \\
15.7 \\
25.2 \\
14.5\end{array}$ & $\begin{array}{r}9.6-15.3 \\
11.4-20.0 \\
20.7-29.8 \\
10.6-18.5\end{array}$ \\
\hline
\end{tabular}

mean compatibility effects are presented in Figure 3. A series of one-sample $t$ tests indicated that nonzero benefits were obtained for auditory distractors in both the easy and the hard conditions. Furthermore, nonoverlapping $95 \%$ confidence intervals indicate that benefits were greater in the hard condition than in the easy condition for auditory distractors and visual distractors. Significant benefits were not obtained for visual distractors for either level of difficulty. Nonzero costs were obtained for visual distractors only in the easy condition. Significant costs were not obtained for auditory distractors for either level of difficulty. Finally, nonzero compatibility effects were found for auditory distractors in both the easy and the hard conditions. Compatibility effects for auditory distractors were larger in the hard condition than in the easy condition. Nonzero compatibility effects were obtained for visual distractors in the easy condition, but not in the hard condition.

\section{Discussion}

This pattern of results and the magnitude of the compatibility effects for visual distractors in Experiment 1 are similar to those obtained by Lavie and Cox (1997). The pattern of results for auditory distractors, however, differs markedly from that obtained for visual distractors. Specifically, significant auditory distractor compatibility effects were found for both easy and hard searches. Furthermore, not only were auditory distractor compatibility effects greater than zero for the hard search condition, these effects were larger than those obtained in the easy search condition for visual or auditory distractors, as is shown in Figure 3. We had expected auditory distractor compatibility effects for easy and hard searches to be similar, since Rees et al. (2001) had shown that distractor perception in one modality was unaffected by task load in another modality.

In addition, significant benefits were obtained for compatible auditory distractors, whereas significant costs were not obtained for incompatible auditory distractors. Previous investigators (Handy \& Mangun, 2000; Handy et al., 2001; Lavie, 1995; Lavie \& Cox, 1997) have not found benefits for compatible visual distractors in any search conditions. In those studies, when significant compatibility effects occurred, they consisted only of costs for incompatible visual distractors. Incompatible auditory distractors in the present study did not appear to interfere with target processing, relative to performance with a neutral distractor. Incompatible visual distractors, however, yielded only significant costs, as similar studies have found (e.g., Lavie \& Cox, 1997). Furthermore, some theorists, such as Pashler (1998), have suggested that attention acts primarily to inhibit nontarget processing. Benefits would be indicative of a form of response priming by compatible auditory distractors that does not occur for compatible visual distractors. Costs are indicative of response competition due to incompatible distractors.

Before accepting the hypothesis that auditory distractors prime responding to visual targets, we must question why incompatible auditory distractors in the present study did not appear to interfere with target processing, relative to performance with a neutral distractor. Perhaps the pattern of costs and benefits obtained for auditory distractors in Experiment 1 occurred because our "neutral" auditory stimuli were not neutral. That is, when the distractor letters $\mathrm{L}$ and $\mathrm{T}$ were presented in a spoken voice, they may have influenced responding. Specifically, these spoken letters may have acted to distract pro- 

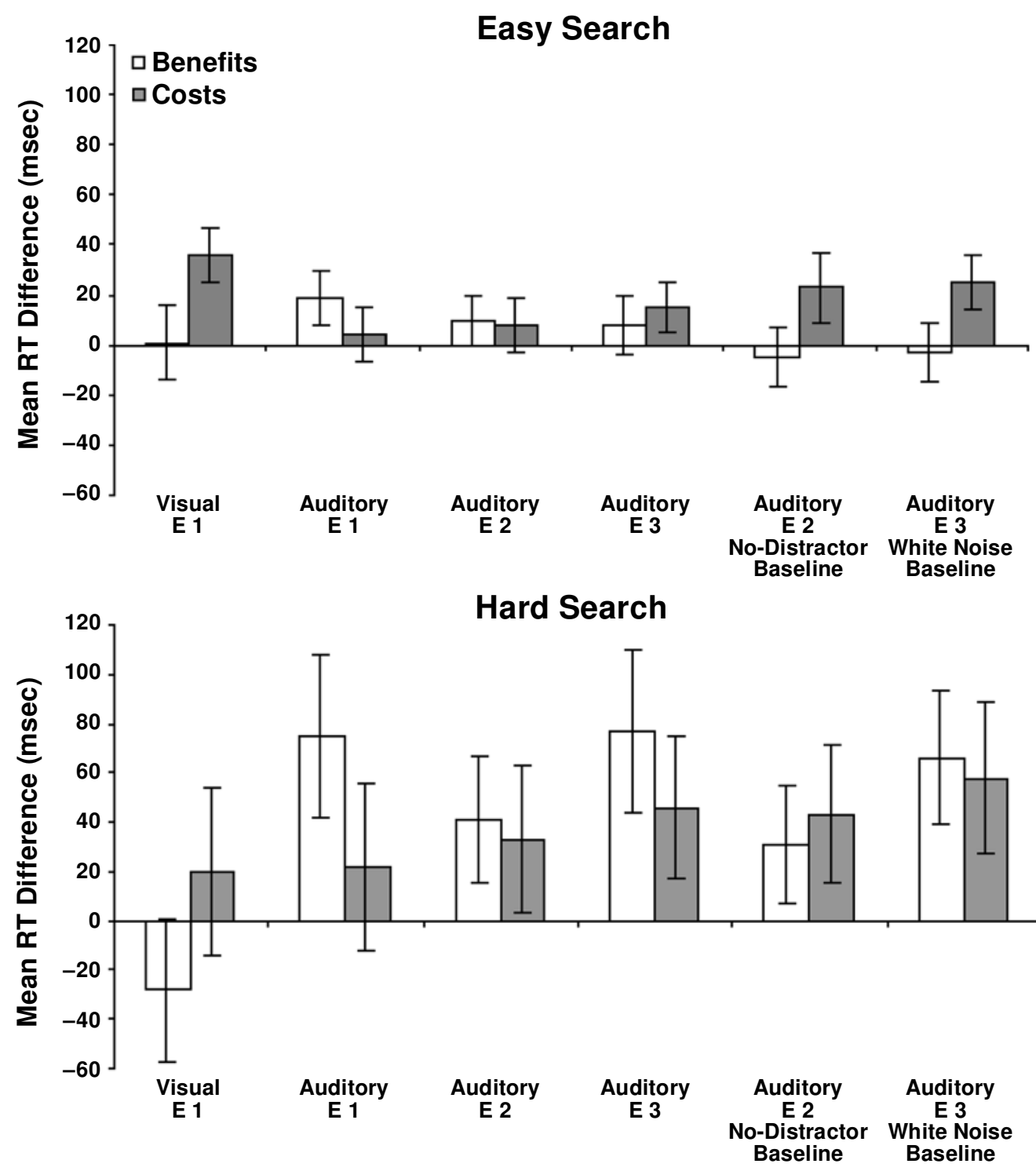

Figure 2. Mean benefits and costs (in milliseconds) for visual and auditory distractors presented for easy and hard searches in Experiments 1-3. Benefit = median reaction time $($ RT $)$ for baseline minus median RT for compatible trials. Cost $=$ median RT for incompatible trials minus median RT for baseline. Unless otherwise noted, baseline was median RT for neutral trials. Error bars $=95 \%$ confidence intervals.

cessing in a way that brought about interference similar to that caused by an incompatible distractor. If this were the case, the neutral distractor comparison in the auditory modality may have indicated benefits, rather than costs, because an improper baseline was used. In fact, the auditory neutral distractors may have negatively impacted processing. Obtained error rates to both auditory neutral and auditory incompatible distractors were relatively high and were greater than those to auditory compatible distractors (see Table 1). This was particularly the case for hard searches, for which the largest benefits were obtained. Error rates and RTs to visual neutral distractors, however, were similar to those obtained for vi- sual compatible distractors, and the obtained results for these conditions replicated those of previous studies (e.g., Lavie \& Cox, 1997). Experiments 2 and 3 were conducted to confirm the task difficulty compatibility effect for auditory distractors and to further investigate costs and benefits by using other baseline conditions.

\section{EXPERIMENT 2}

Although the results of Experiment 1 replicate those of Lavie and Cox (1997) when the distractors were visual, unexpected results were obtained when the distractors were auditory. Experiment 2 was designed to ad- 


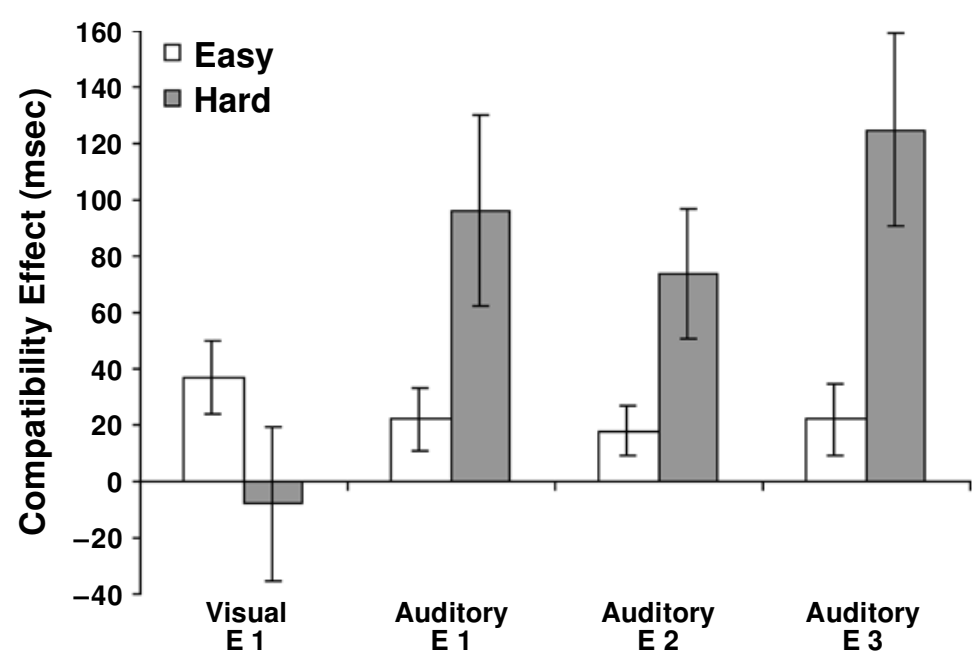

Figure 3. Mean compatibility effects (in milliseconds) for visual and auditory distractors presented for easy and hard searches in Experiments 1-3. Compatibility effect $=$ median reaction time $(\mathrm{RT})$ for incompatible trials minus median RT for compatible trials. Error bars $=95 \%$ confidence intervals.

dress two important issues regarding the effects of auditory distractors. First, we wished to confirm the results of Experiment 1 by replicating the auditory distractor conditions. The auditory distractor compatibility effects differed from those we had hypothesized and were strikingly different from those found for visual distractors in Experiment 1 and in other, previous studies involving visual distractors in which a response competition paradigm was used (e.g., Lavie \& Cox, 1997). Second, we wished to further explore the benefits and costs respectively associated with compatible and incompatible auditory distractors. We questioned why incompatible auditory distractors did not appear to interfere with target processing, relative to performance with a neutral distractor, yet compatible auditory distractors appeared to speed target processing. Incompatible visual distractors yielded only significant costs in Experiment 1, as Lavie and Cox had found. Perhaps when the distractor letters $\mathrm{L}$ and $\mathrm{T}$ were presented in a spoken voice, they did not constitute a bias-free baseline condition and may have had an unknown influence on target processing. To test this possibility in Experiment 2, we included trials during which no distractor was presented, in addition to the compatible, incompatible, and neutral distractor trials presented in Experiment 1 . This allowed us to explore how those distractors designated as neutral in Experiment 1 influenced target processing.

\section{Method}

Participants. Thirty undergraduate students (14 females, 16 males) from a midwest liberal arts college participated on a volunteer basis. Eleven individuals had participated in Experiment 1. Their mean age was 19.6 years $(S D=0.3)$. All the participants had normal or corrected-to-normal near-vision acuity.

Apparatus and Stimuli. The apparatus and stimuli for Experiment 2 were identical to those in Experiment 1, with two exceptions:
Only auditory distractors were presented, and on some trials, no distractor was presented (no-distractor condition).

Design. The independent variables in this experiment were task difficulty (easy or hard) and distractor compatibility (compatible, incompatible, neutral, or no distractor). Compatible, incompatible, and neutral distractor conditions were identical to those in Experiment 1 . The no-distractor condition consisted of the target display presentation without a distractor. As in Experiment 1, all the conditions were fully randomized.

Procedure. The procedure was the same as that in Experiment 1, except that the participants completed three blocks of 96 trials each. The first block was counted as practice and was not used in data analysis. This yielded 24 presentations per condition.

\section{Results}

The results show larger effects of distractor compatibility in hard searches than in easy searches.

We computed median correct RTs and percentage error rates for each participant as a function of search difficulty (easy or hard) and distractor compatibility (compatible, incompatible, neutral, or no distractor). Response latencies below $100 \mathrm{msec}$ or above $2 \mathrm{sec}$ were counted as errors.

We performed a repeated measures ANOVA on the error rate data with two factors: difficulty $(2) \times$ compatibility (4). These data are shown in Table 1. Significant main effects of difficulty $[F(1,29)=95.09, p<.001]$ and of compatibility $[F(3,87)=33.02, p<.001]$ were subsumed in a significant difficulty $\times$ compatibility interaction $[F(3,87)=17.84, p<.001]$. Error rates were lower for easy searches than for hard searches. In addition, when performing hard searches, the participants had higher error rates for incompatible distractors than for any of the other conditions.

We also performed a repeated measures ANOVA on the RT data that are presented in Table 1. Difficulty (2) and compatibility (4) were once again factors. The two-way 
difficulty $\times$ compatibility interaction was significant $[F(3,87)=4.79, p<.004]$. As in Experiment 1 , to further explore how distractor compatibility influences RT, we computed distractor effect magnitudes. In this experiment, however, we had two possible baselines: neutral distractors and no distractors. Hence, we computed two sets of costs and benefits (along with compatibility effects) for each difficulty level. Neutral distractor baseline benefits were computed for each participant by subtracting the median RT for compatible trials from the median RT for neutral trials. Neutral distractor baseline costs were computed by subtracting the median RT for incompatible trials from the median RT for neutral trials. No-distractor baseline benefits were computed by subtracting the median RT for compatible trials from the median RT for nodistractor trials. No-distractor baseline costs were computed by subtracting the median RT for incompatible trials from the median RT for no-distractor trials. Compatibility effects were computed as in Experiment 1. All mean benefits and costs are presented in Figure 2, and mean compatibility effects are presented in Figure 3. A series of one-sample $t$ tests on benefits and costs indicated that for the easy condition, the only nonzero difference was for no-distractor baseline costs. For the hard condition, significantly nonzero costs and benefits were obtained with both neutral distractors and no distractors as baseline. One-sample $t$ tests on the compatibility effects indicated nonzero effects in both the easy and the hard conditions. In addition, the compatibility effect for the hard condition was larger than that for the easy condition.

\section{Discussion}

The results of Experiment 2 replicated those of Experiment 1 in that larger compatibility effects were obtained under hard search conditions than under easy search conditions. In addition, the magnitude of these effects was similar for Experiments 1 and 2. The pattern of costs and benefits, however, differed from that in Experiment 1. Neutral distractor and no-distractor baselines in Experiment 2 yielded similar costs and benefits in the hard search condition, but neutral auditory distractors in Experiment 1 yielded only benefits in hard searches. In Experiment 3, we attempted to further explore the distribution of distractor effects by using yet another baseline condition.

\section{EXPERIMENT 3}

Although the overall magnitude and pattern of compatibility effects were similar for Experiments 1 and 2, when costs and benefits are examined separately, the results of these two experiments are not consistent. In Experiment 3 , rather than presenting trials in which no auditory distractor was presented, as we did in Experiment 2, we presented a burst of white noise as a distractor in one condition. We reasoned that perhaps a noise of any type might have an automatic alerting effect that was distracting. Therefore, we presented a burst of white noise, approximately equal in SPL to the spoken-letter distractors, to determine further how responses to compatible and incompatible distractors would differ. This condition, along with the condition designated as neutral in Experiments 1 and 2, served as a baseline in this experiment and allowed us to again make comparisons of costs and benefits as a function of search difficulty.

\section{Method}

Participants. Thirty undergraduate students (20 females, 10 males) from a midwest liberal arts college participated on a volunteer basis. None of these participants had participated in Experiments 1 or 2 . Their mean age was 18.7 years $(S D=0.2)$. All the participants had normal or corrected-to-normal near-vision acuity.

Apparatus and Stimuli. The apparatus and stimuli for Experiment 3 were identical to those in Experiment 2, with one exception: Auditory distractors were presented on all the trials. In addition to the same compatible, incompatible, and neutral distractors as those presented in Experiment 2, a new type of distractor, white noise, was presented on one fourth of the trials. This distractor consisted of 70-dB white noise presented for $300 \mathrm{msec}$.

Design. The independent variables in this experiment were task difficulty (easy or hard) and distractor compatibility (compatible, incompatible, neutral, or white noise). As in Experiments 1 and 2, all the conditions were fully randomized.

Procedure. The procedure was the same as that in Experiment 2: The participants completed three blocks of 96 trials each. The first block was counted as practice and was not used in data analysis. This yielded 24 presentations per condition.

\section{Results}

The results replicated those of Experiment 2 in that larger distractor effects were obtained in hard searches than in easy searches.

Once again, we computed median correct RTs and percentage error rates for each participant as a function of search difficulty (easy or hard) and distractor compatibility (compatible, incompatible, neutral, or white noise). Response latencies below $100 \mathrm{msec}$ or above $2 \mathrm{sec}$ were counted as errors.

We performed a repeated measures ANOVA on the error rate data with two factors: difficulty $(2) \times$ compatibility (4). These data are shown in Table 1. Significant main effects of difficulty $[F(1,29)=105.03, p<$ $.001]$ and of compatibility $[F(3,87)=13.15, p<.001]$ were subsumed in a significant difficulty $\times$ compatibility interaction $[F(3,87)=22.17, p<.001]$. Error rates were lower for easy searches than for hard searches. In addition, when performing hard searches, the participants had higher error rates for incompatible distractors than for any of the other conditions. There were no other significant effects for the error rate data. This is the same pattern for error rate data as in Experiment 2.

We also performed a repeated measures ANOVA on the RT data that are presented in Table 1. Difficulty (2) and compatibility (4) were again factors. The two-way difficulty $\times$ compatibility interaction was significant $[F(3,87)=16.18, p<.001]$. As in Experiments 1 and 2, to further explore how distractor compatibility influences RT, we computed distractor effect magnitudes. The two possible baselines in this experiment were neu- 
tral distractors and white noise distractors. Hence, we computed two sets of compatibility effects for each difficulty level. Neutral distractor baseline benefits were computed for each participant by subtracting the median RT for compatible trials from the median RT for neutral trials. Neutral distractor baseline costs were computed by subtracting the median RT for incompatible trials from the median RT for neutral trials. White noise baseline benefits were computed by subtracting the median RT for compatible trials from the median RT for white noise trials. White noise baseline costs were computed by subtracting the median RT for incompatible trials from the median RT for white noise trials. Compatibility effects were computed as in Experiments 1 and 2. All mean benefits and costs are presented in Figure 2, and mean compatibility effects are presented in Figure 3 . The pattern of costs and benefits was the same regardless of whether neutral distractor trials or white noise distractor trials were used as baselines. A series of one-sample $t$ tests indicated that in the easy condition, costs, but not benefits, were significantly different from zero. In the hard conditions, significant nonzero differences were found for both costs and benefits. In addition, the magnitude of the costs in the easy condition was significantly smaller than the magnitude of the costs or the benefits in the hard condition. Finally, one-sample $t$ tests on the compatibility effects indicated nonzero effects in both the easy and the hard conditions. As in Experiments 1 and 2 , the compatibility effect for the hard condition was larger than that obtained for the easy condition.

\section{Discussion}

On the whole, the results of Experiment 2 and Experiment 3 were remarkably similar. Benefits and costs, when significantly nonzero, were much smaller for easy searches in Experiments 1-3. Significant and large benefits and costs were consistently obtained across all the experiments for auditory distractors presented during hard searches, with the exception of a failure to obtain significant costs in the hard search condition of Experiment 1 . This exception may have been due to the relatively high error rates to neutral distractors in the hard search condition of that experiment. Unlike the hard search conditions in Experiments 2 and 3, in which error rates to neutral distractors were not significantly different from those to compatible distractors, in Experiment 1, error rates to neutral distractors were higher than those to compatible distractors but did not significantly differ from those to incompatible distractors. It is not clear why performance in the neutral distractor, hard search condition was so poor in Experiment 1. The results of Experiments 2 and 3 suggest that the neutral distractors used in this study do function as a reasonable baseline condition for comparison between compatible and incompatible distractors. Similar benefits and costs were found in Experiment 2 when a no-distractor condition was used as a baseline and in Experiment 3 when a white noise distractor condition was used as a baseline. In addition, the similarity in compatibility effects across the three exper- iments further indicates that the results for the neutral distractors in the hard search condition of Experiment 1 are anomalous.

\section{GENERAL DISCUSSION}

The results of the present study suggest that the perceptual load of a visual task differentially influences the ability to ignore visual and auditory distractors in a response competition paradigm. This difference has important implications for the validity of the perceptual load model and for theories of cross-modal attention.

In Experiment 1, visual distractors influenced easy visual searches, but not hard searches. This outcome fits Lavie's (1995) perceptual load model, in which irrelevant distractor processing is prevented by increasing the load for relevant processing. In fact, our data mirror Lavie and Cox's (1997) findings. The 95\% confidence interval for costs (median RT for incompatible trials minus median RT for neutral trials) for a search set size of one (our easy condition) in the present experiment ranged from 16 to $47 \mathrm{msec}$. Lavie and Cox indicated effects that were between approximately 20 and $30 \mathrm{msec}$ (see Lavie \& Cox, 1997, Figure 2 and Figure 3). In both our study and that of Lavie and Cox, effects of visual distractors for a search set size of six (our hard condition) were not significantly different from zero.

A very different pattern of results was obtained when the distractors were auditory. Significant compatibility effects (median RT for incompatible trials minus median RT for compatible trials) were found for both easy and hard searches in Experiments 1, 2, and 3. In addition, these compatibility effects were larger for hard searches than for easy searches. Benefits and costs for auditory distractors were quite similar in magnitude across the three experiments (see Table 1 for values). Taken together, the results of these three experiments indicate that auditory distractors tend to generally prime responses, with response-compatible auditory distractors producing benefits and response-incompatible auditory distractors producing costs, particularly during hard searches. Visual distractors produced only response competition (costs), when they had any effect at all.

These data appear to strongly suggest that the perceptual load model does not hold cross-modally when targets are visual and distractors are auditory. In fact, our pattern of results is in the direction opposite that which would be expected of the perceptual load model if it applied across modalities, assuming a single processing capacity. The perceptual load model would predict greater distractor interference in the low-load condition, in which spare attentional capacity would automatically be allocated to the processing of distractors. Instead, greater auditory distractor influence occurred under the high-load condition than under the low-load condition. This was the case for both RT and error rate data.

Rees et al. (2001) also have suggested that the perceptual load model does not hold across modalities. Rees et al. (2001) found that when participants performed an 
auditory linguistic task, perception of and brain activation related to an irrelevant visual motion stimulus were unaffected by the perceptual load of the auditory task. They also showed that the perception of the same distractor was influenced by the perceptual load of an unrelated visual task, as they had found in an earlier study (Rees et al., 1997). Under high visual load, perception of irrelevant visual stimuli was reduced, as compared with a low-load situation. Rees et al. (2001) claimed that their results supported the perceptual load model of selective attention, with the caveat that resources are modality specific. Our results, however, show that modalities may not be completely separate. An unexpected finding in the present study was that processing load in one modality influenced processing in another modality, but in the direction opposite that predicted by the perceptual load model (e.g., Lavie \& Tsal, 1994).

There are two major methodological differences between the present study and Rees et al. (2001) that might account for these different patterns of results. First, Rees et al. (2001) manipulated the load of an auditory task, while simultaneously presenting a visual distractor. In our study, the task was visual, whereas the distractors were auditory. Perhaps auditory and visual modalities map onto each other differently. For example, using an exogenous spatial cuing paradigm, Spence and Driver (1997) found that auditory cues influenced the localization of visual stimuli but that visual cues did not influence the localization of auditory stimuli. Such a pattern indicates that the effects of visual attention on auditory stimulus perception may not be the same as those for auditory attention on visual stimulus perception.

Although differences in modalities of task and distractor may have yielded a different outcome, a second, more fundamental difference between the present study and Rees et al. (2001) is a more likely cause for the observed difference in outcomes. Rees et al. (2001) directly measured perception of to-be-ignored stimuli that could not be meaningfully associated with the target task. Perception of task-irrelevant visual motion stimuli was assessed in terms of brain activity, indexed with PET, and in terms of MAE duration. Hence, Rees et al. (2001) studied the influence of varying the perceptual load of a primary task on the perception of irrelevant distractors. In the present study, we utilized a response competition paradigm, which, as Lavie (1997) has stated, allows only an indirect method of assessing the perception of unattended stimuli by measuring the effects of those stimuli on the RT and accuracy to discriminate attended stimuli. A response competition paradigm, however, does directly measure the effect of interference caused by irrelevant stimuli. Determining the extent of such interference was the focus of the present study. Thus, our results are not inconsistent with Rees et al. (2001), who studied a different phenomenon and used a different dependent measure.

A reasonable explanation for the present results may be one that combines the perceptual load model with the possibility of inhibition of cross-modal priming. The findings of Rees et al. (2001) suggest that distractors in one modality are processed regardless of task load in another modality. In the present study, some of the auditory distractors mapped onto responses for the visual modality task. Those distractors that were from the response set could act to prime a response. Folk, Remington, and Johnston (1992), for example, have found that attention can be captured automatically by spatial cues that include properties required for task performance. In addition, Yantis and Johnston (1990) and Fournier, Bowd, and Herbert (2000), using response competition paradigms, found that distractors that match task demands or goals may automatically capture attention. It follows that in the present study, when compatible auditory distractors were presented, the proper response to the target and the response primed by the auditory distractor were the same and RTs to these targets were shorter, as compared with baseline (benefits). When incompatible auditory distractors were presented, however, a conflict occurred between the proper response to the target and the response primed by the auditory distractor, resulting in longer RTs to these targets (costs).

This reasoning is not sufficient, however, to account for compatibility effect size differences between easy and hard searches. Driver and Tipper (1989) showed that both priming and interference can occur at an abstract level of representation - well beyond initial perception of a stimulus. In addition, Handy and Mangun (2000) have suggested that the effects of attention vary across processing stages, depending on the processing performed at a particular stage. In their visual search study, they manipulated spatial expectancy, as well as perceptual load. They found that expectancy, as well as perceptual load, influenced distractor effects. Therefore, they hypothesized that capacity limits may vary throughout the sequence of information processing, and not simply at the level of stimulus perception. In the present study, we cannot address when, in the time course of processing, visual and auditory priming and interference occur. $\mathrm{Fu}-$ ture research may give insight into these processes by varying the onset of distractors relative to the onset of the search array.

In addition, recall that Rees et al. (2001) showed no cross-modal inhibition in the perception of distractors from another modality. However, they did not measure how responding to the perception of distractors in one modality might vary as a function of the perceptual load of performing a task in another modality. A comparison of information from vision and audition obviously would be made well beyond the initial perception of each modality's input. Response activation brought about by distractors in the auditory modality may be inhibited during the processing necessary for the visual search task. If part of the capacity to process a visual task is allocated to inhibit task-relevant, to-be-ignored stimuli from another modality, changes in task load may influence efficacy of this inhibition. Thus, the ability to inhibit this priming may vary inversely with processing 
load. That is, when the perceptual load of the visual task was low (the easy condition in our study), participants had sufficient processing capacity to inhibit the influence of auditory distractors. In this case, benefits and costs were minor or nonsignificant, and compatibility effects were small. When the perceptual load of the visual task was high (the hard condition in our study), capacity to inhibit the influence of the auditory distractors was low. In this case, significant benefits and costs were obtained, and compatibility effects were larger than when the perceptual load was low.

Response priming, rather than interference alone, appears to be a critical cross-modal mechanism in the present study. Significant benefits were obtained when compatible auditory distractors were presented during hard searches in all three experiments reported in the present paper. This pattern differs from that found in Experiment 1 when the distractors and the search task were visual. Similarly, none of Lavie's previous unimodal research on perceptual load indicated significant benefits resulting from compatible distractors (see Lavie, 2001, for a review). Handy et al. (2001) stated that studies of load and distractor interference have not attempted to interpret benefits, because it was not possible to separate effects of feature-based priming and response-based priming. In the present study, however, such a differentiation was possible. When the distractors and the targets were presented in different modalities, feature-based effects could not influence processing. Therefore, any benefits that occurred must have been due to facilitation from priming of responses.

Obtaining significant benefits in any condition of the present study was surprising and prompted Experiments 2 and 3 . The effects of neutral distractors consisting of spoken letters not from the target set were remarkably similar to the effects when no distractor was present (Experiment 2) or when a meaningless auditory distractor was present (white noise in Experiment 3; see Table 1). These results further confirm the hypothesis that targetrelated auditory information acted to prime responses. When auditory distractors were not from the visual target set, they had no more impact on RT and error rate than would presentation of a meaningless auditory distractor or no distractor at all. When the auditory distractor was compatible with the correct visual target letter, responses were faster, and the error rate was generally lower; when the auditory distractor was incompatible with the target letter, responses were generally slower, and the error rate was higher.

Therefore, we propose that Lavie's perceptual load model, with a modification to account for the effects of cross-modal distractors, may account for the present data. Visual attentional capacity may be used for two purposes when distractors are present. First, capacity is utilized to perform the visual search task, and any remaining capacity is automatically allocated to processing visual distractors, as the perceptual load model posits (Lavie \& Tsal, 1994). Second, when auditory distractors are present, processing capacity may be used to inhibit the processing of auditory stimuli that might interrupt visual processing. When capacity is exhausted, visual distractors may be processed less, but auditory distractors may also be inhibited less. Hence, the magnitude of visual distractor effects would be greater in the easy condition than in the hard condition, but the magnitude of auditory distractor effects would be greater in the hard condition than in the easy condition. In sum, just as the processing load of a relevant task determines whether distractors from the same modality are processed, the processing load in one modality determines whether responses to relevant distractors from another modality are inhibited. Note that the perceptual load model (Lavie \& Tsal, 1994) does not include inhibition as a mechanism of selective attention.

The present study also has implications for models of cross-modal attention. Our results indicate some modalityspecific processing for visual and auditory stimuli, as some have suggested. Duncan et al. (1997) arrived at such a conclusion when they presented streams of inputs that contained occasional targets to be identified and recalled. When two auditory input streams (one low and one high voice) or two visual streams (one horizontal and one vertical) were presented simultaneously, the percentage of targets correctly identified was substantially lower than when participants were asked to monitor only one visual or one auditory stream. By comparison, they found that participants could monitor one auditory and one visual input stream simultaneously with no reduction in performance. Duncan et al. showed that when attention was allocated to one modality, it could also be allocated to another modality simultaneously. They concluded that a major component of attentional restriction must be modality specific. Our data do not contradict this conclusion. The participants in Duncan et al.'s study monitored both auditory and visual inputs for targets, whereas the participants in our study were to respond only to visual targets, while ignoring the auditory input. We found in this situation that stimuli could have crossmodal effects that occurred beyond the initial perception of stimuli in each modality. Therefore, there is also some linkage in processing across modalities, as Driver and Spence (1998) have concluded. The present data, however, certainly argue against a conceptualization of attention as centrally limited, with no modality-specific resources (e.g., Kahneman, 1973).

On the basis of Rees et al. (2001), it appears that task load in one modality does not impact processing in another modality when the stimuli in one modality are unrelated to the stimuli in the other modality. On the basis of the present research, we hypothesize that the degree to which auditory information impacts visual processing varies inversely with the perceptual load of current visual processing. In addition, that impact can result in interference with or enhancement of visual processing, but only when the stimuli from one modality map onto the response required for the other modality. Future research 
is necessary to confirm whether this pattern of results holds for visual distractors presented during the processing of auditory targets, as well as across other modalities.

\section{REFERENCES}

Driver, J., \& Spence, C. (1998). Crossmodal attention. Current Opinion in Neurobiology, 8, 245-253.

Driver, J., \& TiPPER, S. P. (1989). On the nonselectivity of "selective" seeing: Contrasts between interference and priming in selective attention. Journal of Experimental Psychology: Human Perception \& Performance, 15, 304-314.

Duncan, J., Martens, S., \& Ward, R. (1997). Restricted attentional capacity within but not between sensory modalities. Nature, $\mathbf{3 8 7}$, 808-810.

ERIKSEN, B. A., \& ERIKSEN, C. W. (1974). Effects of noise letters upon the identification of a target letter in a nonsearch task. Perception \& Psychophysics, 16, 143-149.

Folk, C. J., Remington, R. W., \& Johnston, J. C. (1992). Involuntary covert orienting is contingent on attentional control settings. Journal of Experimental Psychology: Human Perception \& Performance, 18, 1030-1044.

Fournier, L. R., Bowd, C., \& Herbert, R. J. (2000). Interference from multi-dimensional objects during feature and conjunction discriminations. Quarterly Journal of Experimental Psychology, 53A, 191209.

Handy, T. C., \& Mangun, G. R. (2000). Attention and spatial selection: Electrophysiological evidence for modulation by perceptual load. Perception \& Psychophysics, 62, 175-186.

Handy, T. C., Soltani, M., \& Mangun, G. R. (2001). Perceptual load and visuocortical processing: Event-related potentials reveal sensorylevel selection. Psychological Science, 12, 213-218.

Kahneman, D. (1973). Attention and effort. Englewood Cliffs, NJ: Prentice-Hall.

LaVIE, N. (1995). Perceptual load as a necessary condition for selective attention. Journal of Experimental Psychology: Human Perception \& Performance, 21, 451-468.

LAVIE, N. (1997). Visual feature integration and focused attention: Re- sponse competition from multiple distractor features. Perception \& Psychophysics, 59, 543-556.

LAVIE, N. (2001). Capacity limits in selective attention: Behavioral evidence and implications for neural activity. In J. Braun, C. Koch, \& J. L. Davis (Eds.), Visual attention and cortical circuits (pp. 49-68) Cambridge, MA: MIT Press.

Lavie, N., \& Cox, S. (1997). On the efficiency of visual selective attention: Efficient visual search leads to inefficient distractor rejection. Psychological Science, 8, 395-398.

LaVIE, N., \& TSAL, Y. (1994). Perceptual load as a major determinant of the locus of selection in visual attention. Perception \& Psychophysics, 56, 183-197.

Moray, N. (1959). Attention in dichotic listening: Affective cues and the influence of instructions. Quarterly Journal of Experimental Psychology, 11, 56-60.

NAvon, D., \& Gopher, D. (1979). On the economy of the human processing system. Psychological Review, 80, 214-255.

PASHLER, H. E. (1998). The psychology of attention. Cambridge, MA MIT Press.

Rees, G., Frith, C. D., \& Lavie, N. (1997). Modulating irrelevant motion perception by varying attentional load in an unrelated task. Science, 278, 1616-1619.

Rees, G., Frith, C. [D.], \& Lavie, N. (2001). Processing of irrelevant visual motion during performance of an auditory attention task Neuropsychologia, 39, 937-949.

Spence, C., \& Driver, J. (1997). Audiovisual links in exogenous covert spatial orienting. Perception \& Psychophysics, 59, 1-22.

WiCKENS, C. D. (1980). The structure of attentional resources. In R. S Nickerson (Ed.), Attention and performance VIII (pp. 239-257). Hillsdale, NJ: Erlbaum

YANTIS, S., \& JohnSTON, J. C. (1990). On the locus of visual selection Evidence from focused attention tasks. Journal of Experimental Psychology: Human Perception \& Performance, 16, 135-149.

ZELNIKER, T. (1971). Perceptual attenuation of an irrelevant auditory verbal input as measured by an involuntary verbal response in a selectiveattention task. Journal of Experimental Psychology, 87, 52-56.

(Manuscript received August 14, 2002; revision accepted for publication February 14, 2003.) 